\title{
SEARCH FOR SURVIVING COMPANIONS IN TYPE Ia SUPERNOVA REMNANTS
}

\author{
Kuo-Chuan Pan (潘國全) ${ }^{1}$, Paul M. Ricker ${ }^{2}$, and Ronald E. Taam ${ }^{3,4}$ \\ ${ }^{1}$ Physik Department, Universität Basel, Klingelbergstrasse 82, CH-4056 Basel, Switzerland; \\ kuo-chuan.pan@unibas.ch \\ ${ }^{2}$ Department of Astronomy, University of Illinois at Urbana-Champaign, 1002 West Green \\ Street, Urbana, IL 61801, USA; pmricker@illinois.edu \\ ${ }^{3}$ Department of Physics and Astronomy, Northwestern University, 2145 Sheridan Road, \\ Evanston, IL 60208,USA; r-taam@northwestern.edu \\ ${ }^{4}$ Academia Sinica Institute of Astronomy and Astrophysics, P.O. Box 23-141, Taipei 10617, \\ Taiwan; taam@asiaa.sinica.edu.tw
}

\begin{abstract}
The nature of the progenitor systems of type Ia supernovae is still unclear. One way to distinguish between the single-degenerate scenario and double-degenerate scenario for their progenitors is to search for the surviving companions. Using a technique that couples the results from multi-dimensional hydrodynamics simulations with calculations of the structure and evolution of main-sequence- and helium-rich surviving companions, the color and magnitude of main-sequence- and helium-rich surviving companions are predicted as functions of time. The surviving companion candidates in Galactic type Ia supernova remnants and nearby extragalactic type Ia supernova remnants are discussed. We find that the maximum detectable distance of main-sequence surviving companions (helium-rich surviving companions) is $0.6-4 \mathrm{Mpc}(0.4-16 \mathrm{Mpc})$, if the apparent magnitude limit is 27 in the absence of extinction, suggesting that the Large and Small Magellanic Clouds and the Andromeda Galaxy are excellent environments in which to search for surviving companions. However, only five Ia SNRs have been searched for surviving companions, showing little support for the standard channels in the singe-degenerate scenario. To better understand the progenitors of type Ia supernovae, we encourage the search for surviving companions in other nearby type Ia supernova remnants.
\end{abstract}

Subject headings: binaries: close, — methods: numerical, — stars: evolution, —stars: subdwarfs, - supernovae: general, - supernovae: individual (SN 1006, SN 1572, SN 1604, SNR 0509-67.5, SNR 0519-69.0) 


\section{Introduction}

Thermonuclear explosions of carbon-oxygen (CO) white dwarfs (WDs), which lead to Type Ia supernovae (SNe Ia), could be triggered by the merger of two WDs (the double-degenerate scenario (DDS) Iben \& Tutukov 1984; Webbink 1984) or by the accretion of matter from a nondegenerate star through Roche-lobe overflow (the single-degenerate scenario (SDS) Whelan \& Iben 1973; Nomoto 1982). In the SDS, the companion to the CO-WD could be a main-sequence (MS), red giant $(\mathrm{RG})$, or helium-rich $(\mathrm{He})$ star. In the DDS, the companion could be another CO WD or He WD. Based on current studies, both scenarios are not ruled out by observations, but are also not proven by them. If both scenarios lead to SN Ia, it is still unclear which channel(s) in the SDS and DDS dominate(s) the SNe Ia, and bv what fraction Wang \& Han 2012: Hillebrandt et al. 2013; Maoz et al. 2013; Ruiz-Lapuente 2014).

Recent multi-dimensional hydrodynamics simulations of SN impact on the non-degenerate binary companions in the SDS (Marietta et al. 2000; Pakmor et al. 2008; Pan et al. 2010, 2012a; Liu et al. 2012, 2013b) suggest that the companions should survive the SN impact and could be detectable. Therefore, one of the direct methods to distinguish between the SDS and DDS is to search for the surviving companions (SCs) in SN Ia remnants (Ia SNRs).

Pan et al. (2010, hereafter P10) and Pan et al. (2012a, hereafter P12a) examine the effects of a SN impact on the non-degenerate binary companions in the SDS for MS, RG, and He star binary companions via multi-dimensional hydrodynamics simulations. These simulations include the symmetry-breaking effects of orbital motion, rotation of the binary companion, and Roche-lobe overflow (RLOF), all of which allow a better description of SN-driven shock compression, heating, and stripping of SCs. Liu et al. (2012, 2013b) examined a similar SN impact on MS and He stars with the SPH approach using companion models from more sophisticated one-dimensional binary evolutions. However Liu et al. did not study the subsequent post-impact evolution and therefore could not predict the properties of SCs in historical Ia SNRs. On the other hand, Podsiadlowski (2003) and Shappee et al. (2013) examined the evolution of a $1 M_{\odot}$ subgiant and a $1 M_{\odot}$ MS companion with ad hoc prescriptions for energy input and mass stripping without performing detailed hydrodynamical calculations, thereby dramatically overestimating the luminosity of SCs. In Pan et al. (2012b, hereafter P12b) and Pan et al. (2013, hereafter P13), we mapped our detailed three-dimensional hydrodynamical results into a one-dimensional stellar evolution code to simulate the post-impact evolution of MS- and He-SCs and thus provided a more realistic treatment of post-impact evolution.

In this paper, we calculate the time evolution of the magnitudes and colors of our models and discuss the possibility of searching for SDS-SCs in specific nearby Ia SNRs. We present numerical results in $\S 2$ and compare them with searches for SCs in Galactic Ia SNRs in $\S 3$, in the Magellanic Clouds in $\S 4$, and in M31 and M82 in $\S 5$. Finally, we discuss the evidence for the SDS channel for $\mathrm{SNe}$ Ia and present our conclusions in the last section. 


\section{Numerical Results and Predictions}

In this section, we discuss and predict some possible observables of SCs in nearby Ia SNRs and describe differences between SC candidates and unrelated stars in Ia SNRs. In particular, we calculate the colors, magnitudes, effective temperatures, and photospheric radii of MS- and He-SCs as functions of time. We also predict the magnitude and effective temperature changes as functions of time. The linear and rotational velocities of SCs are also discussed. We also suggest upper limits for the $\mathrm{Ni} / \mathrm{Fe}$ contamination in SCs.

\subsection{Colors and Magnitudes of Surviving Companions}

In simulations of MS- and He-SCs, the bolometric luminosity, effective temperature, and photospheric radius are directly determined using the stellar evolution code MESA 1 (Modules for Experiments in Stellar Astrophysics; Paxton et al. 2011, 2013). To facilitate direct comparison with optical observations, the bolometric luminosity is converted to broad band magnitudes. Additional observable quantities such as the strength of absorption lines require the treatment of detailed radiation transfer effects in stellar atmosphere models, which are not considered in this paper.

Given the effective temperature and photospheric radius of a SC, the magnitude of the SC can be estimated under the assumption that the photosphere emits a blackbody radiation spectrum. We have considered several different filters with their corresponding sensitivity functions in this study, including the Johnson-Cousins-Glass UBVIR system and the HST/WFC3 system. The absolute magnitudes are calculated in the $\mathrm{AB}$ magnitude system. For a given extinction, $A_{V}$, the extinction curve can be calculated using the fitting formula in O'Donnell (1994).

The stellar parameters of MS- and He-SC models in our previous work are summarized in Table 1. Figures 1 and 2 illustrate the Hertzsprung-Russell (H-R) diagram in different representations using the $\mathrm{B}$ and $\mathrm{V}$ wavebands and HST wavebands respectively. He-SCs are sdO-like stars that exhibit stronger emission in the $U$ and $B$ bands, while MS-SCs exhibit greater emission in the $V$ and $I$ bands (A-K subgiants). The absolute magnitudes of MS-SCs (He-SCs) span the range $3<M_{V} \lesssim-1\left(4 \lesssim M_{B} \lesssim-4\right)$. The brightest phase of MS-SCs corresponds to Ia SNR ages of $\sim 500-3000 \mathrm{yr}$, which are similar to the ages of most known historic nearby Ia SNRs (see Table 2). Therefore, if these SNRs originated from normal SNe Ia via the SDS MS or the He star channels, SCs should be detectable. In the RG donor channel, almost the entire envelope of a RG should be removed during the SN impact (Marietta et al. 2000; P12a). Therefore, the SC in Ia SNR will no longer be a giant star, but could be a helium degenerate core star with a shallow hydrogen-rich envelope.

\footnotetext{
${ }^{1}$ http://mesa.sourceforge.net
} 
It should be noted that in our calculations, we assume normal SNe Ia with Chandrasekhar mass explosions, and the explosions are initially spherically symmetric. Asymmetric explosions, sub-Chandrasekhar, or super-Chandrasekhar mass explosions may reduce or enhance the evolution of post-impact luminosity (P12b).

\subsection{Rates of Magnitude and Effective Temperature Change}

In addition to comparisons of the colors and magnitudes of SC candidates, the long term variation of magnitude and effective temperature changes is an alternative way to identify the SC. In Figure 1 we see that MS-SCs show rapid luminosity changes but maintain similar effective temperatures in the early few hundred years after an explosion. Therefore, a slight magnitude drop but without color change for a few years after the first observation is expected for MS-SCs. On the other hand, He-SCs show both magnitude and color changes in the first ten years. Figure 3 and Figure 4 show the magnitude and effective temperature variations of our MS- and He-SCs as functions of time. Brighter SC models (Models A, B, and D) have a maximum magnitude change

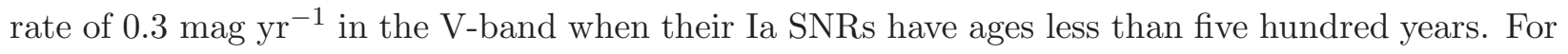
Models C, E, and F, the MS-SCs show a smoother rate of change of magnitude during the first thousand years, but the maximum rate is less than $0.01 \mathrm{mag} \mathrm{yr}^{-1}$, which is very difficult to detect with current optical telescopes. Model G radiates the deposited energy immediately and shows a positive magnitude gradient (gets dimmer). The rate of change of magnitude for Model $\mathrm{G}$ is less than $10^{-4} \mathrm{mag} \mathrm{yr}^{-1}$. The effective temperatures of all our MS-SC models are roughly constants. The highest rate of change is about $200 \mathrm{~K} \mathrm{yr}^{-1}$ for Models $\mathrm{A}, \mathrm{B}$, and $\mathrm{D}$ and $<1 \mathrm{~K} \mathrm{yr}^{-1}$ for other models.

However, the rates of change of magnitude and effective temperature for He-SCs could be notable. All our He-SCs change by $0.5-1 \mathrm{mag} \mathrm{yr}^{-1}$ in the B-band during the first three years and maintain $\sim 0.1-0.3 \mathrm{mag} \mathrm{yr}^{-1}$ over the first decade. Furthermore, He-SCs increase in temperature by $\sim 1,000 \mathrm{~K} \mathrm{yr}^{-1}$ during the first $1-3$ years and then decrease by $-1,000 \sim-4,500 \mathrm{~K} \mathrm{yr}^{-1}$ in the first decade. Therefore, these magnitude and effective temperature changes could be detected in young and nearby Ia SNRs if the SC is a He star.

\subsection{Stellar Velocity and Search Radius}

A SC will have an abnormal speed due to its original orbital speed plus the SN kick. The momentum transfer between the SN ejecta with the donor star will give a kick velocity perpendicular to its orbital velocity. The kick velocity is about $\sim 50-100 \mathrm{~km} \mathrm{~s}^{-1}$ for MS and He star donor channels, and $\lesssim 30 \mathrm{~km} \mathrm{~s}^{-1}$ for the RG donor channel (P10; P12a; Liu et al. 2012, 2013b). By adding the kick velocity to the orbital velocity, the final linear speed at the end of supernova impact is $\sim 130-270 \mathrm{~km} \mathrm{~s}^{-1}$ for MS-SCs, $\sim 440-730 \mathrm{~km} \mathrm{~s}^{-1}$ for He-SCs (see Table 1), and $\lesssim 50 \mathrm{~km} \mathrm{~s}^{-1}$ 
for RG-SCs (P12a). The contribution from the kick velocity could be as high as $30 \%$ in the MS donor channel. Assuming the CO WD has a similar orbital speed (but opposite direction) at the time of explosion and the SNR is moving with this orbital speed, the observational error circle has to include a radius of at least $R_{\mathrm{obs}}>2 v_{\text {linear }} t_{\mathrm{SNR}} \sin i$, where $t_{\mathrm{SNR}}$ is the age of the $\mathrm{SNR}$, and $i$ is the inclination angle. The theoretical estimate of the maximum $v_{\text {linear }}$ is $\sim 1,000 \mathrm{~km} \mathrm{~s}^{-1}$ for the He star donor channel (Wang \& Han 2009).

\subsection{Surface Rotational Speed}

The surface rotational speed of a SC also could be an important signature for SC searches in nearby Ia SNRs. At the time of the SN Ia explosion, the companion should be synchronized by tidal locking and be rapidly rotating, resulting in a surface rotational speed close to $\sim 100 \mathrm{~km} \mathrm{~s}^{-1}$ for MS donors, $\sim 200 \mathrm{~km} \mathrm{~s}^{-1}$ for He star donors, and $\leq 50 \mathrm{~km} \mathrm{~s}^{-1}$ for RG donors (P12a: Meng \& Yang 2011; Liu et al. 2013a). However, because of the angular momentum loss accompanying mass stripping, the surface rotational speed could drop to $\sim 5-30 \mathrm{~km} \mathrm{~s}^{-1}$ for MS donors and $\sim 10-50 \mathrm{~km} \mathrm{~s}^{-1}$ for He star donors after the SN impact (P12b; P13). Once the SN ejecta escape and the $\mathrm{SC}$ reaches hydrostatic equilibrium, the rotational speed could keep decreasing or increasing as a result of post-impact contraction or expansion. If we assume the specific angular momentum is conserved during the post-impact evolution, the surface rotational speed could be in the range from $\lesssim 10 \mathrm{~km} \mathrm{~s}^{-1}$ to $\sim 200 \mathrm{~km} \mathrm{~s}^{-1}$, depending on the amount and depth of SN energy deposition and the age of the SNR (see Figure 11 in P12b and Figure 12 in P13).

In $\mathrm{P} 12 \mathrm{~b}$ and $\mathrm{P} 13$, we have shown that the surface rotation speed will first drop to $\sim 10-$ $30 \mathrm{~km} \mathrm{~s}^{-1}$ after the SN impact and then keep decreasing to $<10 \mathrm{~km} \mathrm{~s}^{-1}$ during the early expansion over a timescale of $10^{2.5}-10^{3} \mathrm{yr}$ for MS-SCs and $10-30 \mathrm{yr}$ for He-SCs. The stellar expansion is due to the release of energy deposited by the SN ejecta. Once the deposited energy has all been released, the envelope of the SC will contract, releasing its gravitational energy. In this phase, the rotational speed may increase up to $10-30 \mathrm{~km} \mathrm{~s}^{-1}$ for MS-SCs and $\sim 200 \mathrm{~km} \mathrm{~s}^{-1}$ for He-SCs. For most of the lifespan of Ia SNRs with ages less than a thousand years, MS-SCs are slowly rotating subgiants but He-SCs are rapidly rotating sdO/B stars.

\subsection{Ejecta Contamination}

Ozaki \& Shigeyama (2006) have suggested that the contamination from SN ejecta may provide observable features in iron absorption lines, which can be used to identify the SCs in Ia SNRs. In $\mathrm{P} 12 \mathrm{a}$, we showed that the amount of Ni/Fe contamination at the surfaces of the SCs is $\sim 10^{-5} M_{\odot}$ for MS star companions, $\sim 10^{-4} M_{\odot}$ for He star companions, and $\sim 10^{-8} M_{\odot}$ for RG companions. At the current stage, our simplified post-impact evolution method cannot predict the strength of any absorption or emission lines during the evolution, but we can provide an order-of-magnitude 
estimate by assuming that these contaminated material are uniformly mixed in the stellar envelope. The estimated upper limit for the nickel-to-hydrogen-plus-helium ratio is about $\sim 10^{-4}$ for MS-SCs, $\sim 10^{-3}$ for He-SCs, and $\sim 10^{-5}$ for RG-SCs (P12a).

\section{Galactic Type Ia SNRs}

The rate of Galactic SNe (including Type I and Type II) is about $2.5_{-0.5}^{+0.8}$ SNe per century, and $\sim 15 \%$ of them are SNe Ia (Tammann et al. 1994). These rates suggest that there should be more than 2,500 SNRs in our Galaxy, and 300 of them are Ia SNRs, if SNRs are recognizable for ages less than $10^{5}$ yrs. However, only $~ 312$ Galactic SNRs have been identified (Ferrand \& Safi-Harb 2012), and only four of them are known as (or likely to be) Ia SNRs $(<2 \%)$. Therefore, a search for a SC in the central region of a SNR could also be an alternative indirect method to identify Ia SNRs among currently-known SNRs. Table 2 shows a summary of the four possible Galactic Ia SNRs (and/or Ia SNR candidates): SN 1006, SN 1572, SN 1604, and RCW 86.

\subsection{SN 1572 (Tycho's SNR)}

The young (442 yr) and nearby $(2.8 \pm 0.4 \mathrm{kpc}$, Ruiz-Lapuente 2004) SNR, Tycho's SNR, has been identified as a normal Ia SNR by its scattered-light echo (Krause et al. 2008). The nonthermal X-ray arc within Tycho's SNR could arise from an interaction between the SN ejecta and mass ejected from the companion star, giving support for the SDS as the progenitor ( $\mathrm{Lu}$ et al. 2011).

Ruiz-Lapuente et al. (2004) found a subgiant star, namely Tycho G, characterized by a high radial velocity $\sim 108 \pm 6 \mathrm{~km} \mathrm{~s}^{-1}$ that could be related to the original orbital speed of the white dwarf companion in the SDS. Kerzendorf et al. (2013, hereafter K13) recently updated this radial velocity to $v_{\mathrm{LSR}} \sim 79 \mathrm{~km} \mathrm{~s}^{-1}$, which is still anomalous for the region. Figure 5 shows our predictions of post-impact conditions of MS-SCs compared with observations by González Hernández et al. (2009, hereafter GH09) and K13. GH09 reported that Tycho G is a subgiant with effective temperature $T_{\text {eff }}=5900 \pm 100 \mathrm{~K}$, surface gravity $\log g=3.85 \pm 0.3 \mathrm{dex}$, and $[\mathrm{Fe} / \mathrm{He}]=-0.05 \pm 0.09 \mathrm{dex}$, using Keck high resolution optical spectra. The distance of Tycho $\mathrm{G}$ is also comparable to that of Tycho's SNR. Using the same data but with a slightly different analysis tool, K13 determined hotter and less luminous characteristics for Tycho $\mathrm{G}\left(T_{\text {eff }}=6000 \pm 100 \mathrm{~K}, \log g=4 \pm 0.3 \mathrm{dex}\right.$, and $[\mathrm{Fe} / \mathrm{H}]=-0.13 \pm 0.13$ dex; see K13 for a more detailed comparison of their results with GH09).

Our Model $\mathrm{E}\left(T_{\text {eff }}=5737 \mathrm{~K}, \log g=3.3 \mathrm{dex}\right.$, and $L=20.2 L_{\odot}$; see Table 3 in $\left.\mathrm{P} 12 \mathrm{~b}\right)$ has a similar effective temperature to that of Tycho $\mathrm{G}$, but is brighter than the luminosity reported by GH09 $\left(L \sim 1.9-7.6 L_{\odot}\right.$, assuming a mass of $\left.1 M_{\odot}\right)$. We note that our Model $\mathrm{E}$ is more massive $\left(M_{E}=1.44 M_{\odot}\right)$ than the mass $\left(1 M_{\odot}\right)$ assumed in GH09 and K13, and the stellar radius and evolution stage are also different with what is suggested in Bedin et al. (2014, hereafter B14). The 
evolution of SCs after the SN explosion depends not only on the amount of mass lost and energy deposition, but also on the depth of energy deposition. Therefore, a less massive companion, different evolutionary stage, asymmetric explosion, or sub-Chandrasekhar mass explosion may better match Tycho G. If Tycho G is indeed similar to our Model E, we predict a magnitude change of $\sim 0.01$ mag for every 10 years (Figure 3).

B14 determined high-accuracy proper motions for 872 SC candidates, including Tycho G, in the central region of Tycho's SNR. They obtained a tangential velocity for Tycho G of $v_{t} \sim$ $64 \pm 11 \mathrm{~km} \mathrm{~s}^{-1}$. Together with the observed radial velocity $\left(80 \mathrm{~km} \mathrm{~s}^{-1}\right)$ of Tycho $\mathrm{G}$ and the average radial velocity $\left(37 \mathrm{~km} \mathrm{~s}^{-1}\right)$ in the direction of Tycho's SNR from the Sun, the linear velocity with respect to the center of Tycho's SNR can be determined to be $\sim 77 \pm 16 \mathrm{~km} \mathrm{~s}^{-1}$, if Tycho G were the $\mathrm{SC}$ (B14). This value is half that of the linear velocities in our models in Table 1. An inclination angle $i \sim 34^{\circ}$, orbital separation $a \sim 26 \pm 12 R_{\odot}$, and orbital period $P=10 \pm 7$ days are also suggested by B14. These progenitor system data are consistent with the spectroscopic observation by GH09 indicating that Tycho G is a G-type subgiant. However, we note that the linear velocity does not equal the orbital speed in the binary system, since the SN kick may contribute up to one-third of its final linear speed (P12a; Liu et al. 2012).

Kerzendorf et al. (2009, hereafter K09) found an upper limit for the rotational speed of Tycho G of $v_{\text {rot }} \sin i \lesssim 7.5 \mathrm{~km} \mathrm{~s}^{-1}$, updated to $v_{\text {rot }} \sin i \lesssim 6 \mathrm{~km} \mathrm{~s}^{-1}$ in K13, causing them to question Tycho G as a SC candidate. P12a, P12b, and Liu et al. (2013a) studied the SN impact and post-impact conditions of MS-like binary companions and found that this discrepancy can be resolved due to the loss of angular momentum during the SN impact and post-impact expansion. However, even when applying the inclination angle in B14, our best model (model E) still has a $v_{r} \sin i=15 \mathrm{~km} \mathrm{~s}^{-1}$, which lies above the upper limit observed by K09 and K13. Similar results have been found in Liu et al. (2013a) as well. Therefore, a less massive or less compact companion model is required to better fit with observations. Fortunately, using the inclination angle and orbital period derived in B14, a low rotational speed of $v_{\text {rot }} \sin i \sim 5.6 \mathrm{~km} \mathrm{~s}^{-1}$ can be calculated by assuming tidal locking, explaining the non-detection of rotational speed in K09 and K13.

On the other hand, Ihara et al. (2007) comments that the absence of an Fe I line feature at $372 \mathrm{~nm}$ in Tycho $\mathrm{G}$ argues against the SC interpretation as there is no evidence for absorption due to the SN ejecta in the stellar spectrum. In contrast, Tycho E shows a strong blueshifted Fe I absorption line without redshifted lines, which implies that it is within the remnant and that its projected position is close to the center of Tycho's SNR, possibly qualifying it as another SC candidate. However, K13 suggests that Tycho E is far behind the SNR and hence the low column density on the receding side of the remnant could explain the lack of redshifted lines. The recent observation of Tycho E reveals an effective temperature $T_{\text {eff }}=5825 \mathrm{~K}$ and surface gravity $\log g=3.4 \mathrm{dex}(\mathrm{K} 13)$, which is also close to our model $\mathrm{E}\left(T_{\mathrm{eff}}=5737 \mathrm{~K}\right.$ and $\left.\log g=3.3 \mathrm{dex}\right)$. However, the low radial velocity $\left(v_{\mathrm{LSR}} \sim 55.91 \pm 0.27 \mathrm{~km} \mathrm{~s}^{-1}\right)$ and large distance $(d \sim 11.2 \pm 7.5 \mathrm{kpc}$, in comparison to $d_{\text {Tycho }} \sim 2.8 \pm 0.8 \mathrm{kpc}$ ) make it less likely to be the $\mathrm{SC}$, although the distance uncertainty is large. Finally, GH09 also have suggested that Tycho E could be a double lined 
binary, which does not provide support for it as a SC. Further detailed observations of these SC candidates are necessary to establish whether any of them is connected to Tycho's SN.

\section{2. $\quad$ SN 1006}

The lack of a compact remnant star, and the amount of iron observed inside the SNR (Hamilton et al. 1997) indicate that SN 1006 was a SN Ia. Measurements of its proper motion indicate that SN 1006 is the closest historical Ia SNR with a distance $d \sim 2.18 \pm 0.08 \mathrm{kpc}$ (Winkler et al. 2003) and an age of $t_{\mathrm{SNR}}=1008 \mathrm{yr}$. The geometric center of the remnant is also well determined in $\mathrm{X}$ ray and radio (Winkler et al. 2003). Furthermore, the foreground extinction is low due to its high Galactic latitude $(b=14.6)$, providing a good environment in which to search for SC candidates.

Searches for SC candidates in SN 1006 have been done using two independent observations by González Hernández et al. (2012, hereafter GH12) and Kerzendorf et al. (2012, hereafter K12). Both teams suggest that there is no evidence for the survival of a $\mathrm{SC}$ in the central region of SN 1006. K12 observed all stars to a limit of $0.5 L_{\odot}(V)$ at the distance of SN 1006 within the central 2 arcminutes. They found no stars as bright as predicted in Marietta et al. (2000) and Podsiadlowski (2003), and no stars show significant rotation. Similar conclusions have been drawn by GH12 using observations of the central 4 arcminutes.

However, we note that the stars B90474 $\left(\log g=3.05 \pm 0.12 \mathrm{~cm} \mathrm{~s}^{-2}, T_{\text {eff }}=5051 \pm 38 \mathrm{~K}\right)$ and B14707 $\left(\log g=3.36 \pm 0.15 \mathrm{~cm} \mathrm{~s}^{-2}, T_{\text {eff }}=5065 \pm 47 \mathrm{~K}\right)$ in GH12 have surface gravities similar to our Model $\mathrm{G}\left(\log g=3.14 \mathrm{dex}, T_{\text {eff }}=5288 \mathrm{~K}\right)$, although with lower effective temperatures (see Figure 5). Furthermore, the star B90474 has a high radial velocity $\left(v_{\text {rad }}=98.10 \pm 1.95 \mathrm{~km} \mathrm{~s}^{-1}\right)$. However, unlike the case of Tycho's SN, SN 1006 lies 500 pc above the Galactic plane, and therefore, the radial velocities of surrounding stars do not exhibit a simple trend. In addition, B90474 has a larger distance uncertainty $(d \sim 4.78 \pm 2.0 \mathrm{kpc})$ and B14707 $(d \sim 1.37 \pm 0.58 \mathrm{kpc})$ lies at a much closer distance than the SNR.

In both GH12 and K12, the authors use the geometric center of the X-ray and radio observations. The error circles included in GH12 and K12 are $4^{\prime}$ and $2^{\prime}$ respectively, corresponding to a SC moving with a speed of $\sim 1,000 \mathrm{~km} \mathrm{~s}^{-1}$ for 2.000 and $1.000 \mathrm{vr}$ at a distance of $2.2 \mathrm{kpc}$. Winkler et al. (2005) have suggested that there is an offset between the geometric center and the center of the iron core. Furthermore, Uchida et al. (2013) and Winkler et al. (2014) have shown that the ejecta distribution in SN 1006 is asymmetric and concentrated in the SE quadrant, suggesting a $5^{\prime} \sim 3.2$ pc offset to the geometric center (Uchida et al. 2013). Winkler et al. (2014) reported that the expansion velocity varies significantly with azimuth $\left(\sim 3000 \mathrm{~km} \mathrm{~s}^{-1}\right.$ in the NW and $7400 \mathrm{~km} \mathrm{~s}^{-1}$ in the SE). Therefore, the error circles used in GH12 and K12 may not include the real explosion center.

On the other hand, the Schweizer-Middleditch star is a subdwarf OB (sdOB) star which is located at the center of SN 1006 and has strong Fe absorption lines (Schweizer \& Middleditch 1980). 
It is relatively bright $(V=16.74, B-V=-0.14)$, with low foreground extinction $(E(B-V)=0.1$; Schweizer \& Middleditch 1980). It is consistent with the He-SC models; however, the presence of redshifted absorption lines due to supernova ejecta suggests that it is more likely a background star (Wu et al. 1983).

\subsection{SN 1604 (Kepler's SNR)}

Kepler's SNR has been identified as a Ia SNR based on X-ray observations of its O/Fe ratio (Reynolds et al. 2007), but its distance is still uncertain $(d \sim 4-6.4 \mathrm{kpc}$; Chiotellis et al. 2012). The interaction of the supernova ejecta with circumstellar material in Kepler's SNR provides evidence that Kepler's SNR may have originated from the SDS in an evolutionary channel consisting of a WD and an asymptotic giant branch (AGB) star (Chiotellis et al. 2012; Burkey et al. 2013). However, the circumstellar medium could be also explained by the stellar wind from a massive progenitor in core-collpase supernova. The low iron mass of $0.01 M_{\odot}$ (Hatsukade et al. 1990) and a progenitor mass of $7 M_{\odot}$ (Hughes \& Helfand 1985) suggest that Kepler's SN may not be a SN Ia.

Recently, Kerzendorf et al. (2014, hereafter K14) have ruled out red giants as SCs due to the lack of bright stars in the central SNR. The observed radial velocity of SC candidates also shows only a small possibility of being associated with the SN explosion. However, 24 stars with $L_{V}>10 L_{\odot}$ at the center of Kepler's SNR have been found by K14, and five of them have $L_{V}>20 L_{\odot}$, perhaps suggesting a relatively higher probability of being a SC. Additional observations are required to test these candidates.

As we pointed out in $\S 2$, nearly all the envelope of a RG should be removed during the SN impact in the RG donor channel, and this is likely to be the case for the AGB donor channel as well. Therefore, the SC in Kepler's SNR would cease to be a AGB star, which would explain the non-detection of a giant star in K14. Follow-up observations and a detailed study of the evolution of the $\operatorname{AGB}(\mathrm{RG})$ channel in the SDS and its SCs will be necessary in order to understand the progenitor system of Kepler's SNR.

\section{4. $\quad$ RCW 86}

RCW 86 is the oldest known Galactic SN Ia. It was recently identified as a Ia SNR by Williams et al. (2011) and Yamaguchi et al. (2011), who estimate that the integrated Fe-K emission corresponds to a total Fe mass of about $1 M_{\odot}$. Its possible association with $\mathrm{SN} 185$ is somewhat dubious since it would imply the age of RCW 86 is 1,829 yr. The large size of its radius at an estimated distance of $\sim 2.5 \mathrm{kpc}$ (Williams et al. 2011) (or $d \sim 2.8 \mathrm{kpc}$; Rosado et al. 1996) suggests a very high shock speed $\left(\sim 7,800 \mathrm{~km} \mathrm{~s}^{-1}\right)$ or a much older age. One explanation is that it originated in a cavity explosion (Vink et al. 1997; Badenes et al. 2007), although cavity explosions are more common in core-collapse SNe. Hence, the connection between RCW 86 and SN 185 has yet to be 
established. In addition, the derived ambient density $\left(0.075 \mathrm{~cm}^{-3}\right)$ found by Yamaguchi et al. (2011) suggests that an unusually low-density cavity surrounds the SNR. This can be understood either as resulting from the existence of a strong stellar wind from the progenitor itself or as an outflow from the nearby OB association discovered by Westerlund (1969). If RCW 86 was a member of this group, the age of this group may place some constraints on the delay-time of this SN Ia, although the ages of these OB stars are still unknown. The wind-blown bubble scenario by Williams et al. (2011) suggests that RCW 86 originated in the SDS.

Therefore, a search for SC candidates in RCW 86 seems reasonable. However, the two expansion fronts in the southwest and the northeast make it difficult to measure the center of the explosion (K14). In our calculations, all MS-SC models reach the brightest phase $\left(>100 L_{\odot}\right)$ and highest effective temperature at around $500-3000 \mathrm{yr}$, depending on the model. Therefore the nearby distance and low extinction $\left(A_{V} \sim 1.7\right.$; Leibowitz \& Danziger 1983) make it easier to distinguish SC from unrelated stars.

\section{Type Ia SNRs in the Magellanic Clouds}

The Magellanic Clouds (MCs) are excellent environments to search for SCs for several reasons. Ia SNRs in the Large Magellanic Cloud (LMC) and Small Magellanic Cloud (SMC) are at known distances, $50 \mathrm{kpc}$ and $60 \mathrm{kpc}$, that are sufficiently close to detect SCs with HST based upon the results in $\S 2$. The MCs are nearly face-on galaxies, minimizing confusion along the line of sight (van der Marel \& Cioni 2001; Subramanian \& Subramaniam 2012). The foreground Galactic extinction is low, and the internal extinction of the MCs is modest (Haschke et al. 2011). Finally, the star formation history (SFH) and metallicity of the MCs are different than those of the Milky Way (Harris \& Zaritsky 2004, 2009; Maoz \& Badenes 2010), allowing the possibility of testing SN Ia progenitors in different type of galaxies (Wang et al. 2013).

Ten Ia SNRs have been reported to lie in the LMC and four in the SMC (see Table 2). Since only four Galactic Ia SNRs have been identified, these fourteen Ia SNRs provide a larger sample of Ia SNRs to search for SCs. In addition, the distances of Ia SNRs in the LMC and SMC are known more accurately than for Galactic Ia SNRs, which reduces the uncertainties in comparing observations with the theoretical predictions.

Recently, the voungest Ia SNR in the LMC. SNR 0509-67.5. has been studied by Schaefer \& Pagnotta (2012). SNR 0509-67.5 formed from a SN 1991T-like SN Ia $\sim 400$ yrs ago. The observation of the central region of SNR 0509-67.5 within a radius of $1.43^{\prime \prime}$, corresponding to a distance of $0.36 \mathrm{pc}$ from the center of SNR 0509-67.5, shows no star brighter than $V=26.9$ $\left(M_{V}=8.4\right)$ in this region. This result rules out all standard SDS channels and suggests that SNR 0509-67.5 originated in the DDS (Schaefer \& Pagnotta 2012).

Edwards et al. (2012) studied the central sources within an error circle of $4.7^{\prime \prime}$ radius about the center the $\sim 600$ yr old Ia SNR 0519-69.0 using HST. They found 27 MS stars brighter than 
$m_{V}=22.7$ magnitude. This result requires the progenitor of SNR 0519-69.0 to arise from either the DDS or the SDS with a supersoft source. We point out that Edwards et al. (2012) found a sub-giant star in SNR 0519-69.0 $\left(V=20.78 \pm 0.01 \mathrm{mag}\right.$, and $\left.V-H_{\alpha}=0.35 \pm 0.01\right)$. Although this star is consistent with our model $\mathrm{G}$ star $\left(V=20.7 \mathrm{mag}\right.$, and $V-H_{\alpha}=0.35$; the black line in Figure 5), it lies close to the edge of the possible error circle, which suggests that it may not be the SC of SNR 0519-69.0 unless the explosion was asymmetric.

\section{Other Nearby Galaxies}

Besides the LMC and SMC, other nearby galaxies can provide samples that probe diverse environments for SCs. In particular, the metallicities and SFHs in other nearby galaxies differ from those of the Milky Way and Magellanic Clouds, which could affect pre-SN conditions, the SN Ia explosion itself, and, potentially, the post-impact evolution.

In Section 2, we have shown that our predicted MS-SCs (He-SCs) span a range of absolute magnitude $3<M_{V} \lesssim-1\left(4 \lesssim M_{B} \lesssim-4\right)$ in F555W (F438W). By using HST/WFC3 (with U, B, $\mathrm{V}$, and I filters), the limiting magnitude with $\mathrm{S} / \mathrm{N}$ of 10 for point sources is $\sim 27$ with a one hour exposure, and $\sim 29$ for a 10 hour exposure, giving maximum distance moduli of $24-28$ and $23-31$ respectively. This corresponds to a maximum detectable distance of $0.6-4 \mathrm{Mpc}(0.4-16 \mathrm{Mpc})$ if there is no extinction. However, as extinction varies from galaxy to galaxy, the maximum detectable distance would be smaller in extreme cases.

Given that He-SCs reach their maximum brightness at 10 - 30 yr after SN explosions and then fade within $\sim 100 \mathrm{yr}(\mathrm{P} 13)$, their $B$ magnitudes are likely less than 1 for historic Ia SNRs. This yields a distance limit of $d_{\mathrm{SN}, \max } \lesssim 0.4 \mathrm{Mpc}$. However, the short timescale $(10-30 \mathrm{yrs})$ of their luminous phase provides the possibility to detect slow transitions of their brightness at the locations of recent nearby SNe Ia with $d_{\mathrm{SN}, \max }<16 \mathrm{Mpc}$. We note that the timescale for the decay of the light curve from the supernova may take longer than the luminous phase of the SC and, hence, that the maximum detectable distance could be smaller.

SNRs in nearby galaxies, including the Andromeda Galaxy (M31 or NGC 224; Blair et al. 1981; Sasaki et al. 2012), NGC 300 (Millar et al. 2012), M82 (Fenech et al. 2010). NGC 4214 (Dopita et al. 2010), NGC 5204, NGC 5585, NGC 6946, M81, and M101(Matonick \& Fesen 1997), have been studied recently. Therefore, Ia SNRs in these galaxies should be targeted for SC searches. However, it is unclear whether these SNRs are Ia SNRs.

\subsection{Andromeda Galaxy (M31)}

More than 26 SNRs (including Type I and Type II) have been identified in the Andromeda

Galaxy at $0.79 \mathrm{Mpc}$ by Blair et al. (1981) and Sasaki et al. (2012). M31 has a distance modulus 
of 24.5, which makes it possible to detect all of our MS-SCs and most He SCs using HST/WFC3. The Panchromatic Hubble Andromeda Treasury (PHAT) multi-cycle program has observed about one third of M31 using HST (Dalcanton et al. 2012). Its UVIS data reached a magnitude limit of $\sim 25$ in the F275W and F336W bands; ACS data reach maximum depths of $\sim 28$ magnitudes in $\mathrm{F} 475 \mathrm{~W}$ and $\sim 27$ magnitudes in $\mathrm{F} 814 \mathrm{~W}$ in the uncrowded outer disk. In these same regions, WFC3/IR data reach maximum depths of $\sim 26.5$ and $\sim 25.5$ in F110W and F160W, respectively.

Sasaki et al. (2012) have listed 26 X-ray SNRs and 20 X-ray SNR candidates in M31 based on their X-ray, optical, and radio emission, which is the most recent complete list of X-ray SNRs in M31. Therefore, using their list together with PHAT's data, it may be possible to identify SC candidates.

SN 1885a (S Andromedae) is a subluminous SN Ia in M31 van den Bergh 2002; Fesen et al. 2007). Its young age and the small size of its remnant make it easier to search for a SC. However, the surrounding light is dominated by the bulge of M31, making SC searches difficult unless the $\mathrm{SC}$ is overluminous.

\subsection{Recent nearby SNe Ia}

SN 2014j was discovered by Stephen Fossey and his students on January 21, 2014 and reported as a SN Ia on January 22, 2014 (Fossev et al. 2014; Cao et al. 2014). SN 2014j is the closest SN Ia $(d \sim 3.5 \pm 0.3 \mathrm{Mpc}$; Karachentsev \& Kashibadze 2006) discovered in the past 42 years. The next close one is SN 1972e (in NGC 5253) with a distance of 4.64 \pm 0.7 Mpc (della Valle \& Melnick 1992). SN 2011fe is also a nearby SN Ia discovered in the modern astronomical era. However, the distance of SN 2011fe (in M101; $d \sim 6.4$ Mpc, Shappee \& Stanek 2011) is above our predicted maximum distance for MS-SCs. A search for SCs in SN 2011fe will be very challenging. Furthermore, the emptiness of the surrounding medium (Chomiuk et al. 2012) and the lack of pre-explosion optical and X-ray source (Liu et al. 2012) suggest a DDS progenitor for SN 2011 fe.

SN 2014j is almost twice as close as SN 2011fe, and the distance (3.5 Mpc) is within our predicted detection limit $(4 \mathrm{Mpc})$ for MS-SCs, though the distance is close to the limit. HST archival observations do not rule out SDS progenitors such as recurrent novae or some classical novae (Williams et al. 2014; Elias-Rosa et al. 2014). However, even when the SNR becomes transparent, any MS-SC would need a few hundred years to become bright enough to be detected by HST. If the $\mathrm{SC}$ is a He-SC, a bright luminous OB-like star is expected to be observed in the next few decades.

\section{Conclusions}

We have determined the colors and magnitudes of seven MS- and four He-SC models based on our stellar evolutionary calculations (P12b, P13) and predicted their rates of change as functions 
of time. We have also studied the linear and rotational speeds of SCs and the potential ejecta contamination of SCs. Comparisons of the model predictions for SCs with the Galactic SNe 1572 and 1006 are presented, assuming all the candidate stars have the same distance as their host SNRs. In particular, it is found that both Tycho E $\left(T_{\text {eff }}=5825 \mathrm{~K}, \log g=3.4\right.$ dex $)$ and Tycho $\mathrm{G}$ $\left(T_{\text {eff }}=5900 \pm 100 \mathrm{~K}, \log g=3.85 \pm 0.3 \mathrm{dex}\right)$ approximately fit our predicted MS-SC Model E $\left(T_{\text {eff }}=5737 \mathrm{~K}, \log g=3.3 \mathrm{dex}\right)$, although there are small discrepancies (K13). Furthermore, two sub-giants in the error circle of SN 1006 are also found to have magnitudes consistent with our MS SC Model G $\left(T_{\text {eff }}=5288 \mathrm{~K} . \log q=3.14 \mathrm{dex}\right)$. but have lower effective temperatures (González Hernández et al. 2012). However it should be noted that the uncertainties in the distances of these $\mathrm{SC}$ candidates are large, and that the derived effective temperatures and surface gravities are not consistent in different papers. In addition to the Galactic Ia SNRs, a sub-giant in the SNR 0519-69.0 is consistent with the magnitude and color of our Model G, but the projected position in the SNR is too far from the center (Edwards et al. 2012). If such a candidate is a confirmed SC, then the original orbital speed (plus SN kick) would be much higher than expected unless the explosion was asymmetric. Although higher orbital speeds would be expected for He SCs, there is no evidence for such a candidate.

Based on the current sample of SNe Ia companion searches, it is more likely that most have originated from the DDS or peculiar SDS channels. Peculiar SDS channels such as the M-dwarf channel (Wheeler 2012) or the spin up/down channel (Justham 2011: Di Stefano et al. 2011; Di Stefano \& Kilic 2012) may explain the non-detection of SC candidates in Ia SNRs. To obtain a better understanding of the progenitor systems of SN Ia, we encourage further SC searches in other Galactic or nearby extragalactic Ia SNRs. Unlike Galactic Ia SNRs, which have large distance uncertainties, the distances of extragalactic Ia SNRs are relatively well-known. We predict that the maximum detectable distance of MS-SCs (He-SCs) is $0.6-4 \mathrm{Mpc}(0.4-16 \mathrm{Mpc})$, if the apparent magnitude limit is 27 with no extinction, suggesting that the LMC, SMC, and M31 are excellent targets in which to search for SCs. Furthermore, we also predict He-SCs will not only show high luminosity and effective temperature (luminous OB-like stars), but also show a rate of magnitude

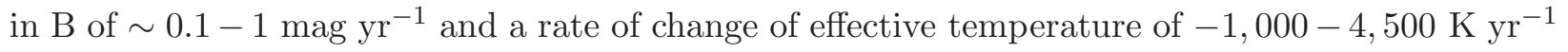
in the first decade since explosion. Future observations of He-SC candidates should look into their magnitude and color changes as well.

Similar analysis can be applied to core-collapse (CC) SNe as well. The observed high fraction of $\mathrm{SN} \mathrm{Ib/c} \mathrm{cannot} \mathrm{be} \mathrm{explained} \mathrm{by} \mathrm{single} \mathrm{stellar} \mathrm{evolution,} \mathrm{suggesting} \mathrm{that} \mathrm{a} \mathrm{high} \mathrm{fraction} \mathrm{of} \mathrm{CCSNe}$ were in binary systems (Smith et al. 2011; Kochanek 2009). However, the ejected-mass-and-energy and companion types and separations are very different from those in SN Ia. In addition, the companion does not need to be in Roche lobe overflow at the time of explosion. Therefore, the evolution of SCs in CCSNe could be very different and vary from case to case. The impact of CCSNe on binary companions and their subsequent evolution are important future work in this area. 
We thank the anonymous referee for his/her valuable comments and suggestions. KCP thanks You-Hua Chu for useful discussions about Ia SNRs in the LMC. This work was supported by the Computational Science and Engineering (CSE) fellowship at the University of Illinois at UrbanaChampaign, by the Theoretical Institute for Advanced Research in Astrophysics (TIARA) in the Academia Sinica Institute of Astronomy and Astrophysics (ASIAA), and by the European Research Council (ERC) grant FISH. The FLASH simulations presented here were carried out using the NSF XSEDE Ranger system at the Texas Advanced Computing Center under allocation TGAST040034N. FLASH was developed largely by the DOE-supported ASC/Alliances Center for Astrophysical Thermonuclear Flashes at the University of Chicago.

\section{REFERENCES}

Badenes, C., Hughes, J. P., Bravo, E., \& Langer, N. 2007, ApJ, 662, 472

Bedin, L. R., Ruiz-Lapuente, P., González Hernández, J. I., et al. 2014, MNRAS, 439, 354

Blair, W. P., Kirshner, R. P., \& Chevalier, R. A. 1981, ApJ, 247, 879

Borkowski, K. J., Reynolds, S. P., Green, D. A., et al. 2010, ApJ, 724, L161

Borkowski, K. J., Reynolds, S. P., Hwang, U., et al. 2013, ApJ, 771, L9

Burkey, M. T., Reynolds, S. P., Borkowski, K. J., \& Blondin, J. M. 2013, ApJ, 764, 63

Cao, Y., Kasliwal, M. M., McKay, A., \& Bradley, A. 2014, The Astronomer's Telegram, 5786, 1

Carlton, A. K., Borkowski, K. J., Reynolds, S. P., et al. 2011, ApJ, 737, L22

Chiotellis, A., Schure, K. M., \& Vink, J. 2012, A\&A, 537, A139

Chomiuk, L., Soderberg, A. M., Moe, M., et al. 2012, ApJ, 750, 164

Dalcanton, J. J., Williams, B. F., Lang, D., et al. 2012, ApJS, 200, 18

della Valle, M., \& Melnick, J. 1992, A\&A, 257, L1

Di Stefano, R., Voss, R., \& Claeys, J. S. W. 2011, ApJ, 738, L1

Di Stefano, R., \& Kilic, M. 2012, ApJ, 759, 56

Dopita, M. A., Calzetti, D., Maíz Apellániz, J., et al. 2010, Ap\&SS, 330, 123

Edwards, Z. I., Pagnotta, A., \& Schaefer, B. E. 2012, ApJ, 747, L19

Elias-Rosa, N., Greggio, L., \& Botticella, M. T. 2014, The Astronomer's Telegram, 5849, 1

Fenech, D., Beswick, R., Muxlow, T. W. B., Pedlar, A., \& Argo, M. K. 2010, MNRAS, 408, 607 
Ferrand, G., \& Safi-Harb, S. 2012, Advances in Space Research, 49, 1313

Fesen, R. A., Höflich, P. A., Hamilton, A. J. S., et al. 2007, ApJ, 658, 396

Fossey, J., Cooke, B., Pollack, G., Wilde, M., \& Wright, T. 2014, Central Bureau Electronic Telegrams, 3792, 1

González Hernández, J. I., Ruiz-Lapuente, P., Filippenko, A. V., et al. 2009, ApJ, 691, 1

González Hernández, J. I., Ruiz-Lapuente, P., Tabernero, H. M., et al. 2012, Nature, 489, 533

Green, D. A., \& Gull, S. F. 1984, Nature, 312, 527

Green, D. A., Reynolds, S. P., Borkowski, K. J., et al. 2008, MNRAS, 387, L54

Hamilton, A. J. S., Fesen, R. A., Wu, C.-C., Crenshaw, D. M., \& Sarazin, C. L. 1997, ApJ, 481, 838

Harris, J., \& Zaritsky, D. 2004, AJ, 127, 1531

Harris, J., \& Zaritsky, D. 2009, AJ, 138, 1243

Haschke, R., Grebel, E. K., \& Duffau, S. 2011, AJ, 141, 158

Hatsukade, I., Tsunemi, H., Yamashita, K., et al. 1990, PASJ, 42, 279

Hillebrandt, W., Kromer, M., Röpke, F. K., \& Ruiter, A. J. 2013, Frontiers of Physics, 8, 116

Hughes, J. P., \& Helfand, D. J. 1985, ApJ, 291, 544

Iben, I., Jr., \& Tutukov, A. V. 1984, ApJS, 54, 335

Ihara, Y., Ozaki, J., Doi, M., et al. 2007, PASJ, 59, 811

Justham, S. 2011, ApJ, 730, L34

Karachentsev, I. D., \& Kashibadze, O. G. 2006, Astrophysics, 49, 3

Kerzendorf, W. E., Schmidt, B. P., Asplund, M., et al. 2009, ApJ, 701, 1665

Kerzendorf, W. E., Schmidt, B. P., Laird, J. B., Podsiadlowski, P., \& Bessell, M. S. 2012, ApJ, 759,7

Kerzendorf, W. E., Yong, D., Schmidt, B. P., et al. 2013, ApJ, 774, 99

Kerzendorf, W. E., Childress, M., Scharwächter, J., Do, T., \& Schmidt, B. P. 2014, ApJ, 782, 27

Kochanek, C. S. 2009, ApJ, 707, 1578

Krause, O., Tanaka, M., Usuda, T., et al. 2008, Nature, 456, 617 
Leibowitz, E. M., \& Danziger, I. J. 1983, MNRAS, 204, 273

Liu, J., Di Stefano, R., Wang, T., \& Moe, M. 2012, ApJ, 749, 141

Lu, F. J., Wang, Q. D., Ge, M. Y., et al. 2011, ApJ, 732, 11

Liu, Z. W., Pakmor, R., Röpke, F. K., et al. 2012, A\&A, 548, A2

Liu, Z.-W., Pakmor, R., Röpke, F. K., et al. 2013a, A\&A, 554, A109

Liu, Z.-W., Pakmor, R., Seitenzahl, I. R., et al. 2013b, ApJ, 774, 37

Marietta, E., Burrows, A., \& Fryxell, B. 2000, ApJS, 128, 615

Maoz, D., Mannucci, F., \& Nelemans, G. 2013, arXiv:1312.0628

Maoz, D., \& Badenes, C. 2010, MNRAS, 407, 1314

Matonick, D. M., \& Fesen, R. A. 1997, ApJS, 112, 49

Meng, X., \& Yang, W. 2011, Science in China: Physics, Mechanics and Astronomy, 54, 2296

Millar, W. C., White, G. L., \& Filipovic, M. D. 2012, Serbian Astronomical Journal, 184, 19

Nomoto, K. 1982, ApJ, 257, 780

O’Donnell, J. E. 1994, ApJ, 422, 158

Ozaki, J., \& Shigeyama, T. 2006, ApJ, 644, 954

Pakmor, R., Röpke, F. K., Weiss, A., \& Hillebrandt, W. 2008, A\&A, 489, 943

Pan, K.-C., Ricker, P. M., \& Taam, R. E. 2010, ApJ, 715, 78

Pan, K.-C., Ricker, P. M., \& Taam, R. E. 2012a, ApJ, 750, 151

Pan, K.-C., Ricker, P. M., \& Taam, R. E. 2012b, ApJ, 760, 21

Pan, K.-C., Ricker, P. M., \& Taam, R. E. 2013, ApJ, 773, 49

Paxton, B., Bildsten, L., Dotter, A., et al. 2011, ApJS, 192, 3

Paxton, B., Cantiello, M., Arras, P., et al. 2013, ApJS, 208, 4

Podsiadlowski, P. 2003, arXiv:astro-ph/0303660

Reynolds, S. P., Borkowski, K. J., Hwang, U., et al. 2007, ApJ, 668, L135

Reynolds, S. P., Borkowski, K. J., Green, D. A., et al. 2008, ApJ, 680, L41

Rosado, M., Ambrocio-Cruz, P., Le Coarer, E., \& Marcelin, M. 1996, A\&A, 315, 243 
Ruiz-Lapuente, P. 2004, ApJ, 612, 357

Ruiz-Lapuente, P. 2014, arXiv:1403.4087

Ruiz-Lapuente, P., Comeron, F., Méndez, J., et al. 2004, Nature, 431, 1069

Sasaki, M., Pietsch, W., Haberl, F., et al. 2012, A\&A, 544, A144

Schaefer, B. E., \& Pagnotta, A. 2012, Nature, 481, 164

Schweizer, F., \& Middleditch, J. 1980, ApJ, 241, 1039

Shappee, B. J., \& Stanek, K. Z. 2011, ApJ, 733, 124

Shappee, B. J., Kochanek, C. S., \& Stanek, K. Z. 2013, ApJ, 765, 150

Smith, N., Li, W., Filippenko, A. V., \& Chornock, R. 2011, MNRAS, 412, 1522

Subramanian, S., \& Subramaniam, A. 2012, ApJ, 744, 128

Tammann, G. A., Loeffler, W., \& Schroeder, A. 1994, ApJS, 92, 487

Uchida, H., Yamaguchi, H., \& Koyama, K. 2013, ApJ, 771, 56

van den Bergh, S. 2002, AJ, 123, 2045

van der Marel, R. P., \& Cioni, M.-R. L. 2001, AJ, 122, 1807

Vink, J., Kaastra, J. S., \& Bleeker, J. A. M. 1997, A\&A, 328, 628

Vink, J. 2012, A\&A Rev., 20, 49

Wang, B., \& Han, Z. 2009, A\&A, 508, L27

Wang, B., \& Han, Z. 2012, New A Rev., 56, 122

Wang, X., Wang, L., Filippenko, A. V., Zhang, T., \& Zhao, X. 2013, Science, 340, 170

Webbink, R. F. 1984, ApJ, 277, 355

Westerlund, B. E. 1969, AJ, 74, 879

Wheeler, J. C. 2012, ApJ, 758, 123

Whelan, J., \& Iben, I., Jr. 1973, ApJ, 186, 1007

Williams, B. J., Blair, W. P., Blondin, J. M., et al. 2011, ApJ, 741, 96

Williams, S. C., Darnley, M. J., Bode, P. A., et al. 2014, The Astronomer's Telegram, 5824, 1

Winkler, P. F., Gupta, G., \& Long, K. S. 2003, ApJ, 585, 324 
Winkler, P. F., Long, K. S., Hamilton, A. J. S., \& Fesen, R. A. 2005, ApJ, 624, 189

Winkler, P. F., Williams, B. J., Reynolds, S. P., et al. 2014, ApJ, 781, 65

Wu, C.-C., Leventhal, M., Sarazin, C. L., \& Gull, T. R. 1983, ApJ, 269, L5

Yamaguchi, H., Koyama, K., \& Uchida, H. 2011, PASJ, 63, 837

Zaritsky, D., Harris, J., Thompson, I. B., \& Grebel, E. K. 2004, AJ, 128, 1606 
Table 1. The MS and He progenitor models

\begin{tabular}{lccccc}
\hline \hline \multicolumn{1}{c}{ Model } & $M_{\mathrm{b}}^{\dagger}\left(M_{\odot}\right)$ & $R_{\mathrm{b}}\left(R_{\odot}\right)$ & $M_{\mathrm{a}}^{\ddagger}\left(M_{\odot}\right)$ & $R_{\mathrm{a}}\left(R_{\odot}\right)$ & $v_{\text {linear }}\left(\mathrm{km} \mathrm{s}^{-1}\right)$ \\
\hline A (MS) & 1.88 & 1.25 & 1.64 & 3.87 & 179 \\
B (MS) & 1.82 & 1.50 & 1.65 & 4.76 & 179 \\
C (MS) & 1.82 & 2.63 & 1.56 & 7.61 & 136 \\
D (MS) & 1.63 & 1.19 & 1.43 & 3.42 & 188 \\
E (MS) & 1.59 & 1.42 & 1.44 & 3.91 & 191 \\
F (MS) & 1.55 & 1.97 & 1.30 & 4.09 & 143 \\
G (MS) & 1.17 & 0.79 & 0.93 & 4.45 & 271 \\
HeWDa (He) & 0.697 & 0.091 & 0.656 & 0.390 & 734 \\
HeWDb (He) & 0.803 & 0.158 & 0.748 & 0.565 & 550 \\
HeWDc (He) & 1.007 & 0.194 & 0.962 & 0.809 & 509 \\
HeWDd (He) & 1.206 & 0.231 & 1.126 & 1.041 & 446 \\
\hline
\end{tabular}

Note. - Seven MS progenitor models (A-G) and four He progenitor models (HeWDa-d) are considered in this study.

${ }^{\dagger}$ The mass $\left(M_{\mathrm{b}}\right)$ and radius $\left(R_{\mathrm{b}}\right)$ for different progenitors at the time of the SN explosion.

$\ddagger$ The initial relaxed hydrostatic mass $\left(M_{\mathrm{a}}\right)$ and radius $\left(R_{\mathrm{a}}\right)$ for different progenitor stars after SN explosion. 
Table 2. Type Ia SNRs in the Milky Way and Magellanic Clouds

\begin{tabular}{|c|c|c|c|}
\hline Name & Age (yrs) & Distance (kpc) & Radius (pc) \\
\hline $\mathrm{RCW} 86(\mathrm{SN} 185 ?)^{\dagger}$ & $>1,800$ & $\sim 2.3-2.8(\mathrm{MW})$ & $\sim 15$ \\
\hline SN 1006 & 1,007 & $2.2 \pm 0.08(\mathrm{MW})$ & 9.3 \\
\hline SN 1572 (Tycho's) & 441 & $2.8 \pm 0.8(\mathrm{MW})$ & 3.8 \\
\hline SN 1604 (Kepler's) & 409 & $\sim 6(\mathrm{MW})$ & 3 \\
\hline B0509-67.5 & $400 \pm 50$ & $50(\mathrm{LMC})$ & 3.6 \\
\hline N103B & $1,000-2,000$ & 50 (LMC) & 3.6 \\
\hline B0519-69.0 & $600 \pm 200$ & $50(\mathrm{LMC})$ & 3.9 \\
\hline DEM L71 & $\sim 4,500$ & 50 (LMC) & 8.6 \\
\hline B0548-70.4 & $\sim 7,000$ & $50(\mathrm{LMC})$ & 12.5 \\
\hline DEM L316A & $?$ & $50(\mathrm{LMC})$ & 15 \\
\hline В0534-69.9 & $\sim 10,000$ & $50(\mathrm{LMC})$ & 16 \\
\hline DEM L238 & $10,000-15,000$ & $50(\mathrm{LMC})$ & 21 \\
\hline DEM L249 & $10,000-15,000$ & $50(\mathrm{LMC})$ & 23 \\
\hline B0454-67.2 & $\sim 30,000$ & $50(\mathrm{LMC})$ & 27 \\
\hline IKT 4 & $?$ & $60(\mathrm{SMC})$ & $\sim 12$ \\
\hline IKT 5 & $?$ & $60(\mathrm{SMC})$ & 15 \\
\hline IKT 25 & $?$ & $60(\mathrm{SMC})$ & 18 \\
\hline DEM S128 & $?$ & $60(\mathrm{SMC})$ & 26 \\
\hline
\end{tabular}

Note. - Four Galactic (MW) Ia SNRs (SN 1006, SN 1572, SN 1604, and RCW 86) and fourteen Ia SNRs in the LMC and SMC that are listed in Vink (2012).

${ }^{\dagger}$ References: Westerlund (1969); Rosado et al. (1996), and Williams et al. (2011). 

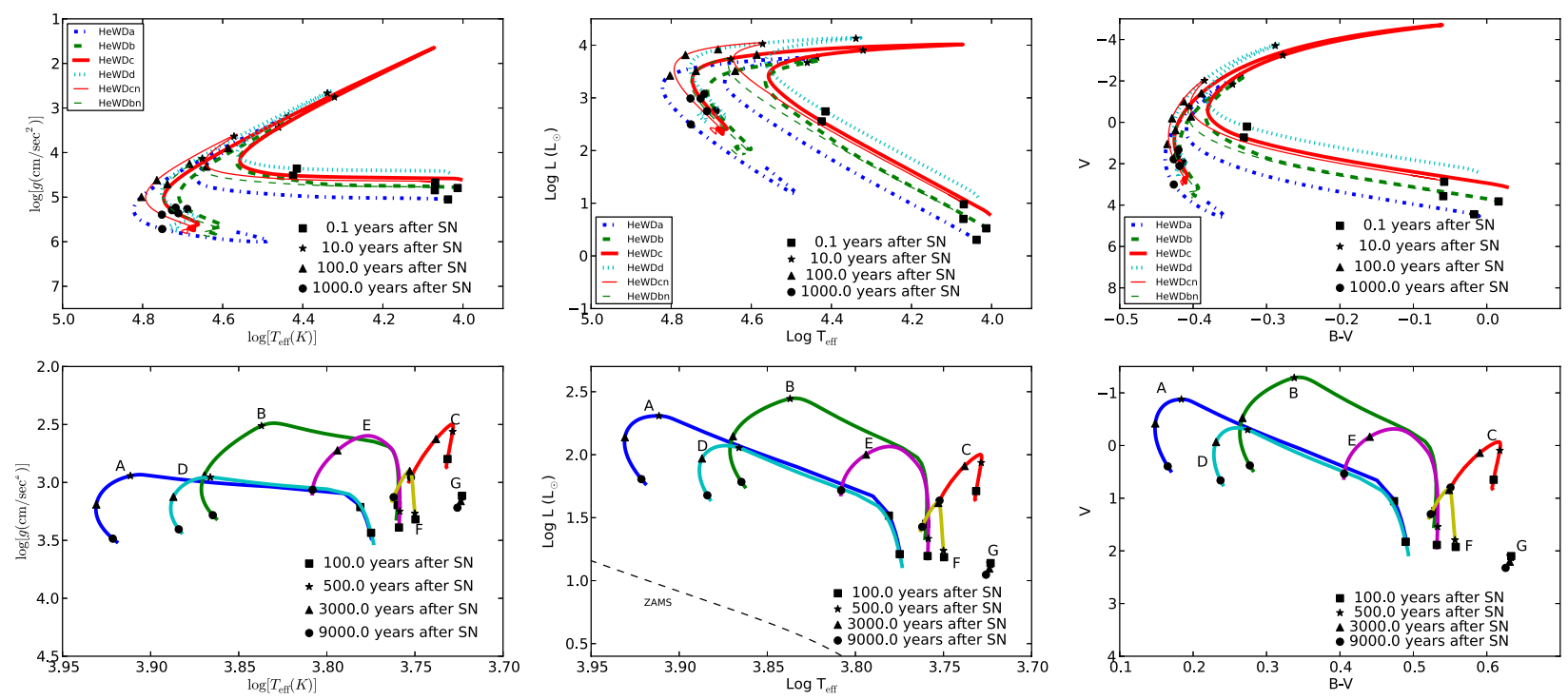

Fig. 1.- Evolutionary tracks in H-R diagrams for He- (upper panels) and MS-SCs (lower panels) using different representations. Color lines indicate different progenitor companions in Table 1. Different symbols show the evolutionary stage at different times. 

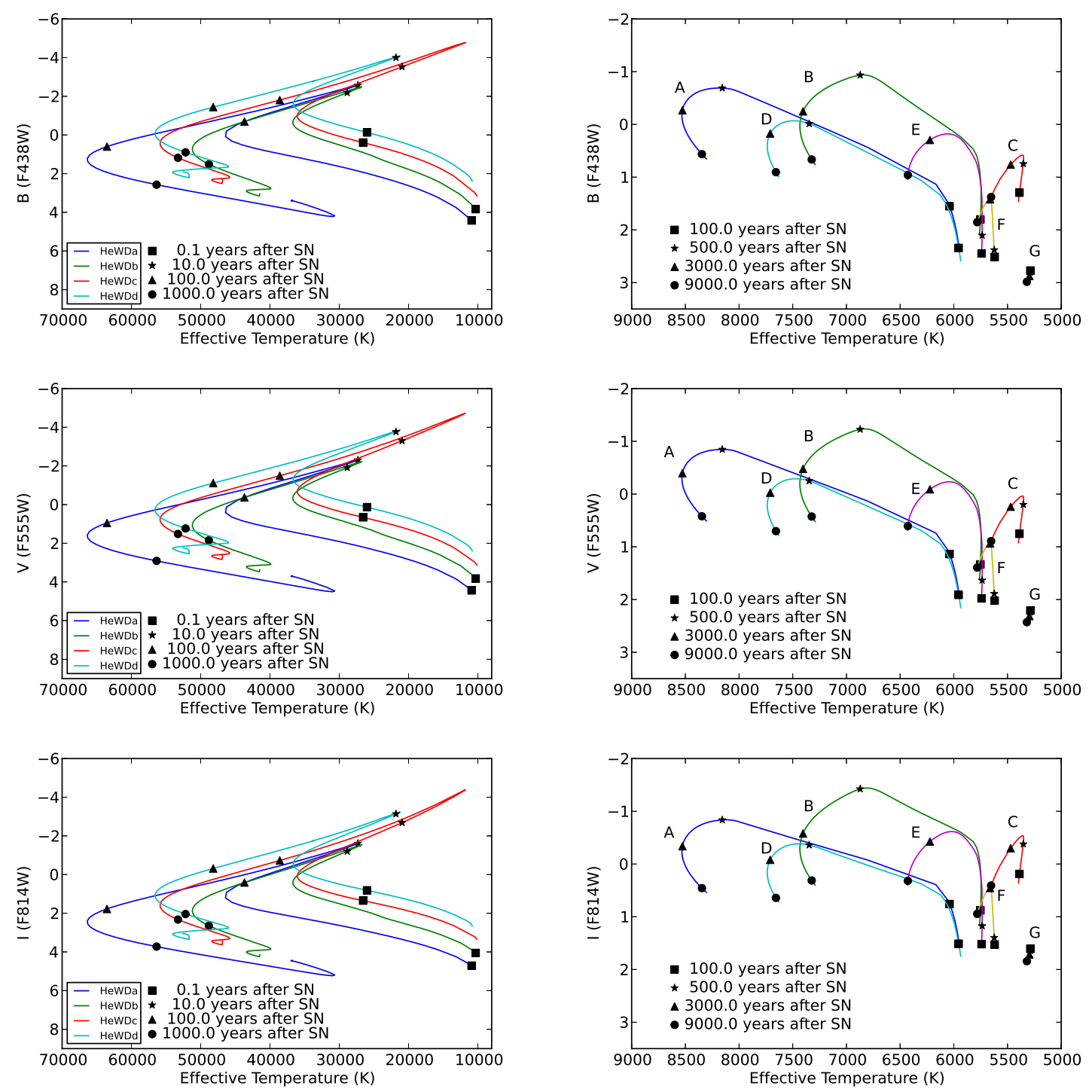

Fig. 2.- Similar to Figure 1 but for different HST/WFC3 band magnitude vs. effective temperature. 

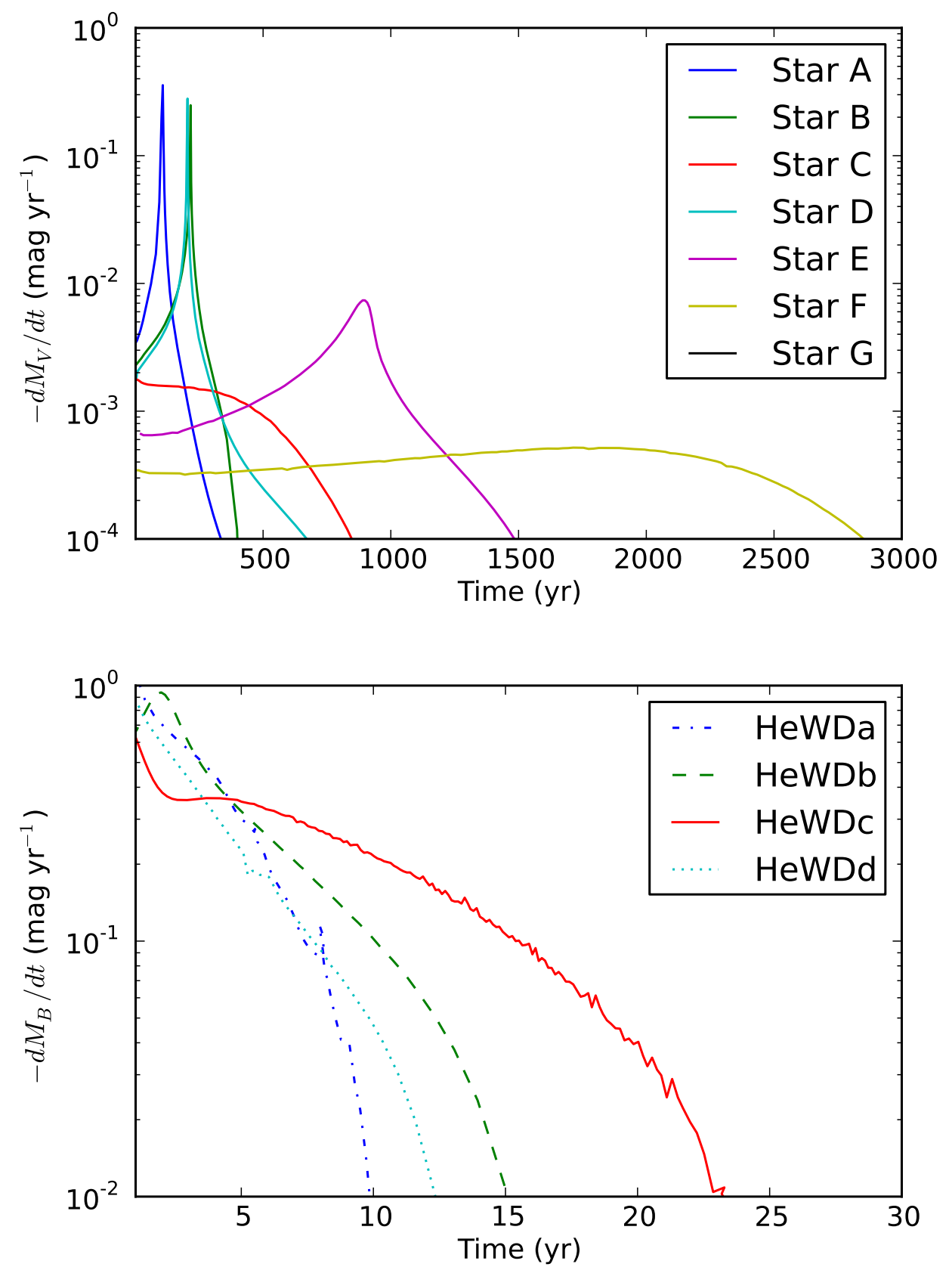

Fig. 3.- Time derivative of magnitude change as functions of time for MS- (top) and He-SCs (bottom). The magnitude of Star G is increased in time and has a magnitude gradient $\left(d M_{v} / d t<\right.$ $10^{-4}$ ), and therefore not shown in the figure. 

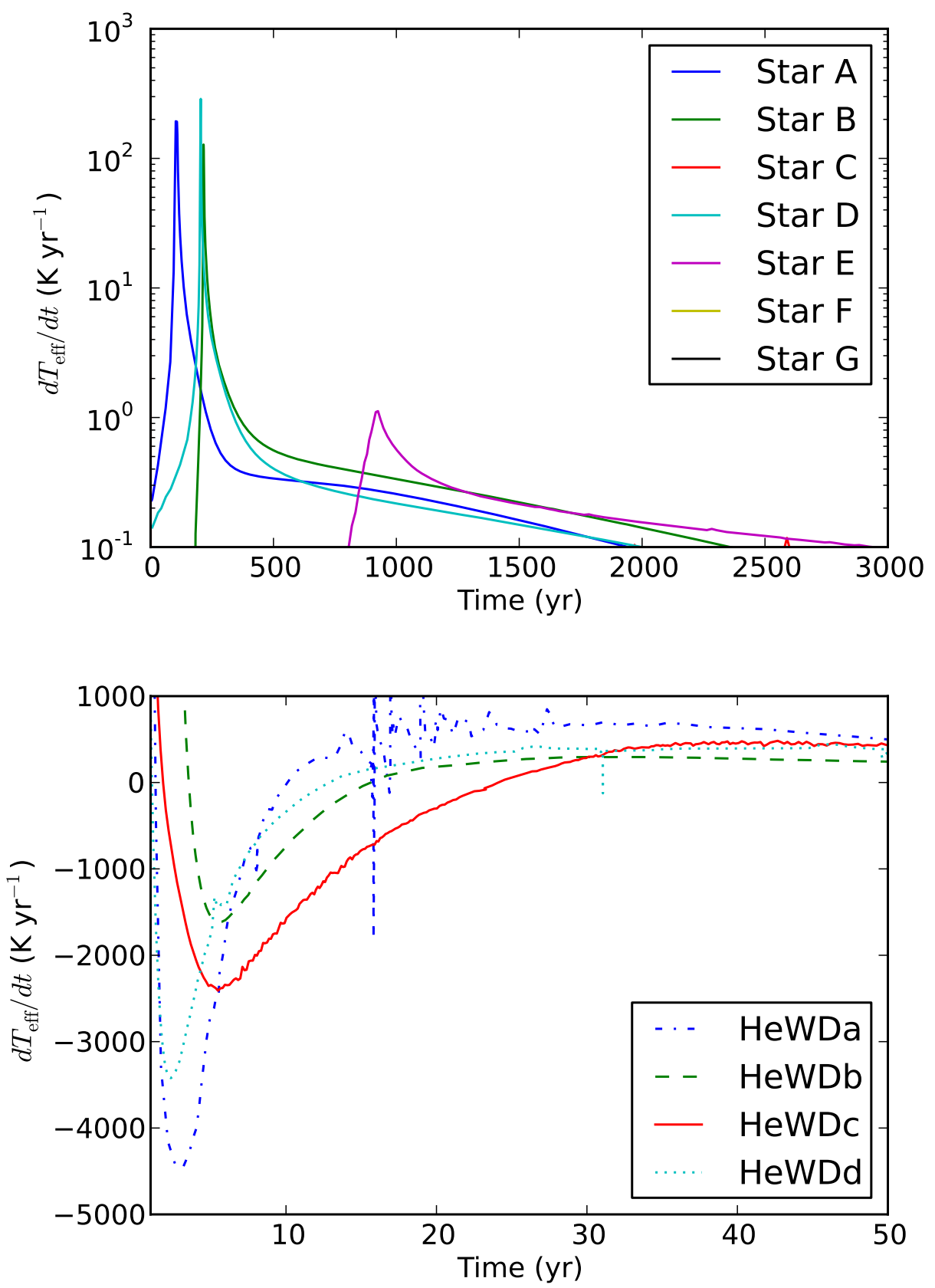

Fig. 4.- Similar to Figure 3 but for time derivative of effective temperature. Model C, F, and G have change rate less than $10^{-1} \mathrm{~K} \mathrm{yr}^{-1}$. 

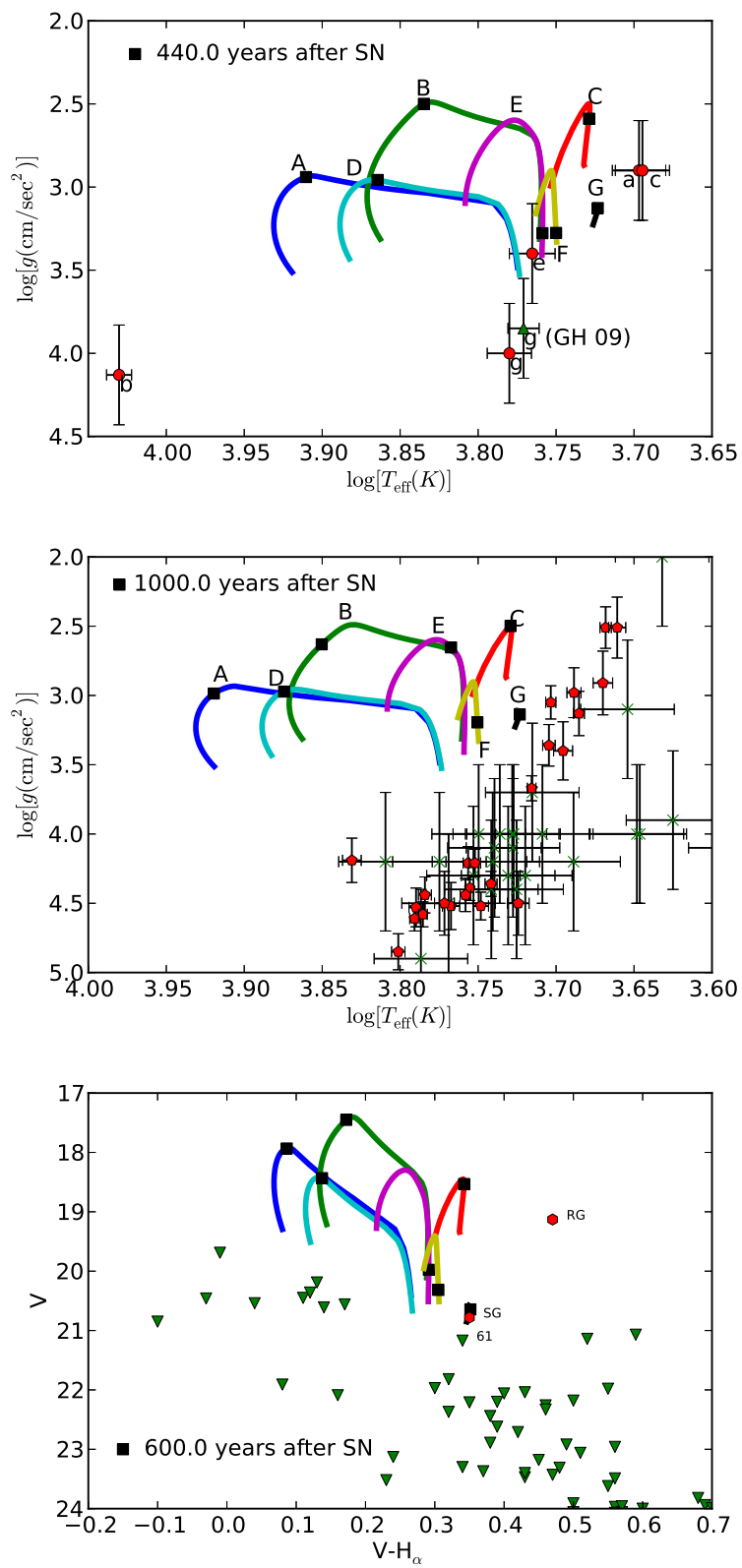

Fig. 5. - Evolutionary tracks in effective temperature vs. surface gravity diagrams and H-R diagram of SC candidates in SN 1572 (top), SN 1006 (middle), and SNR 0519-69.0 (bottom). Color lines indicate the simulated evolutionary tracks for different SC models. Black squares represent the conditions at the ages of Ia SNRs. For SNR 0519-69.0, an extinction of $A_{V}=0.42$ is adopted in our SC calculations (Zaritskv et al. 2004). Note that the ages of historical recorded supernovae (SN 1572, SN 1006) are very accurate, but the ages of SNRs in LMC are less accurate. Fortunately, the early evolution of MS-SCs and late evolution of He-SCs after a hundred years are relatively slow. Other symbols denote the observational conditions of SC candidates from different papers (SN 1572: green up-triangles, González Hernández et al. 2009 and red circles, Kerzendorf et al. 2013; SN 1006: red pentagons, González Hernández et al. 2012 and green stars, Kerzendorf et al. 2012; and SNR 0519-69.0: green down-triangles and red circles, Edwards et al. 2012). 